\title{
The Plasma-Lyte 148 versus Saline (PLUS) statistical analysis plan: a multicentre, randomised controlled trial of the effect of intensive care fluid therapy on mortality
}

Laurent Billot, Rinaldo Bellomo, Martin Gallagher, David Gattas, Naomi E Hammond, Diane Mackle, Sharon Micallef, John Myburgh, Leanlove Navarra, Manoj Saxena, Colman Taylor, Paul J Young, and Simon Finfer, on behalf of the PLUS Study investigators and the ANZICS Clinical Trials Group

This article outlines the statistical analysis plan for the Plasma-Lyte 148 versus Saline (PLUS) study, which is a prospective multicentre, parallel-group, concealed, blinded, randomised controlled trial to determine whether fluid resuscitation and therapy with a balanced crystalloid solution (Plasma-Lyte 148) decreases 90day mortality in critically ill patients requiring fluid resuscitation compared with the same treatment using $0.9 \%$ sodium chloride (saline). ${ }^{1}$

This study is endorsed by the Australian and New Zealand Intensive Care Society Clinical Trials Groups (ANZICS CTG). The approach of publication of a statistical analysis plan before the analysis of data has been used for previous randomised controlled trials conducted by the ANZICS $\mathrm{CTG},{ }^{2}$ including the Crystalloid versus Hydroxyethyl Starch Trial (CHEST), ${ }^{3}$ the Augmented versus Routine Approach to Giving Energy Trial (TARGET), ${ }^{4}$ and the Intensive Care Unit Randomised Trial Comparing Two Approaches to Oxygen Therapy (ICU-ROX). ${ }^{5}$ The use of a pre-specified statistical analysis plan in the PLUS study demonstrates that the study is being conducted with a high degree of transparency and should reduce the risk of analysis bias arising from knowledge of the study findings emerging during analysis of the study data. 2,6

The statistical analysis plan for the PLUS study was developed by the study statistician (LB), the chief investigator $(\mathrm{SF})$, and the project manager (SM), and approved by the PLUS study Management Committee, and released on a pre-print server ${ }^{7}$ before completion of study recruitment.

\section{Study overview}

\section{Design and setting}

The PLUS study is a prospective, multicentre, parallel-group, concealed, blinded, randomised controlled trial, which will be conducted in 40-50 intensive care units (ICUs) in Australia and New Zealand. The PLUS study will test the hypothesis

\begin{abstract}
Background and objective: The Plasma-Lyte 148 versus Saline (PLUS) study is a prospective, multicentre, parallelgroup, concealed, blinded, randomised controlled trial comparing the effect of Plasma-Lyte 148 versus $0.9 \%$ sodium chloride (saline) for fluid resuscitation and other fluid therapy on 90-day mortality among critically ill adults requiring fluid resuscitation. The original target for recruitment was 8800 participants, which was reduced to 5000 participants following the onset of the coronavirus disease 2019 (COVID-19) pandemic in 2020. This article describes the statistical analysis plan for the PLUS study.

Methods: The statistical analysis plan was developed by the study statistician, chief investigator, and project manager, and was approved by the Management Committee before unblinding. The plan describes in detail the analysis of baseline characteristics, process measures, and outcomes, including covariate adjustments, subgroup analyses, missing data handling, and sensitivity analyses.
\end{abstract}

Results and conclusions: A statistical analysis plan for the PLUS study was developed. This pre-specified plan accords with high quality standards of internal validity and should minimise future analysis bias.

Crit Care Resusc 2021; 23 (1): 24-31

that in a heterogeneous population of critically ill adults, random assignment to Plasma-Lyte 148 for intravascular volume resuscitation and crystalloid fluid therapy in the ICU results in different 90-day all-cause mortality compared with random assignment to $0.9 \%$ saline for the same treatment.

The study was prospectively registered on ClinicalTrials.gov (NCT02721654) and the protocol has been published previously. ${ }^{1}$ 


\title{
ORIGINAL ARTICLES
}

\section{Population}

All patients who are admitted to one of the study ICUs will be screened for study eligibility. Patients who fulfil all of the inclusion criteria and none of the exclusion criteria will be eligible for the study (Table 1).

\section{Randomisation and study treatment}

Permuted block randomisation with variable block sizes, stratified by site will occur via a password-protected, secure website. Following successful randomisation, each patient will be assigned a unique patient study number and will be assigned to receive either Plasma-Lyte 148 or $0.9 \%$ saline (blinded study treatment). Study participants, treating clinicians, study investigators and data collectors will be blinded to treatment allocation.

Each study participant will receive either Plasma-Lyte 148 or $0.9 \%$ saline alone for all resuscitation episodes and for all compatible intravenous crystalloid therapy while in the ICU (for up to 90 days). Other crystalloid fluids may be used as carrier fluids for the infusion of any drug for which either Plasma-Lyte 148 or $0.9 \%$ saline is considered incompatible; in such instances, $5 \%$ glucose will be used whenever possible to minimise exposure to open-label Plasma-Lyte 148 and $0.9 \%$ saline. Aside from the study treatment,

\section{Table 1. Eligibility criteria}

\begin{abstract}
Inclusion criteria
- The patient will receive fluid resuscitation, defined as a bolus of fluid prescribed to be administered over one hour or less to increase or maintain intravascular volume; that is, in addition to maintenance fluids, or specific fluids used to replace nonphysiological fluid losses
\end{abstract}

- The patient is expected to be in the ICU the day after tomorrow

- The patient is not expected to be well enough to be eating tomorrow

- An arterial or central venous catheter is in situ, or placement is imminent as part of routine management

- Both Plasma-Lyte 148 and $0.9 \%$ saline are considered equally appropriate for the patient

- The requirement for fluid resuscitation is supported by at least one of seven pre-specified clinical signs:

- heart rate $>90$ beats per minute

- systolic blood pressure $<100 \mathrm{mmHg}$

- mean arterial pressure $<75 \mathrm{mmHg}$

- central venous pressure $<10 \mathrm{mmHg}$

- pulmonary artery wedge pressure $<12 \mathrm{mmHg}$

- capillary refill time $>1$ second

- urine output $<0.5 \mathrm{~mL} / \mathrm{kg}$ for at least one hour

\section{Exclusion criteria}

- Age $<18$ years

- Patients who have received more than $500 \mathrm{~mL}$ of fluid resuscitation (as defined above) prescribed in the ICU during this current ICU admission

- Patients transferred directly from another ICU who have received more than $500 \mathrm{~mL}$ of fluid resuscitation (as defined above) during that ICU admission

- Contraindication to either study fluid (eg, previous allergic reaction to Plasma-Lyte 148)

- Patients admitted to the ICU with specific fluid requirements (eg, the treatment of burns, after liver transplantation surgery, for correction of specific electrolyte abnormalities)

- Patients with traumatic brain injury or those considered at risk of developing cerebral oedema

- Patients in whom death is deemed imminent and inevitable

- Patients with an underlying disease process with a life expectancy of $<90$ days

- Patients in whom it is unlikely the primary outcome can be ascertained

- Known or suspected pregnancy

- Patients who have previously been enrolled in PLUS

ICU = intensive care unit; PLUS = Plasma-Lyte 148 versus Saline study. 


\section{ORIGINAL ARTICLES}

Table 2. Secondary outcomes

- Mean and peak serum creatinine concentration during the first 7 days

- Maximum post-randomisation increase from baseline in serum creatinine in ICU during the index hospital admission

- Proportion of patients newly treated with renal replacement therapy up to 90 days after randomisation

- Proportion of patients treated with and duration of treatment with vasoactive drugs defined as a cardiovascular SOFA score of 2,3 or 4

- Duration of mechanical ventilation in the ICU

- Length of stay and all-cause mortality at ICU discharge

- Length of stay and all-cause mortality at 28 days

- Length of stay and all-cause mortality at hospital discharge

- Quality of life assessed at 6 months after randomisation*

- Health services use during the 6 months after randomisation ${ }^{\dagger}$

ICU = intensive care unit; PLUS = Plasma-Lyte 148 versus Saline study; SOFA = Sequential Organ Failure Assessment. * Quality of life data will be published separately from other data described here and a separate plan for analysis of these data will be developed. $†$ Health services data will be published separately from other data described here and a separate plan for analysis of these data will be developed.

patient management will be otherwise unaffected, and the treating clinicians will be free to provide whatever medical care is deemed best and necessary for the patient.

\section{Outcomes}

The primary outcome is death from all causes within 90 days after randomisation. The secondary outcomes are shown in Table 2.

\section{Sample size}

The study was originally designed to recruit 8800 participants to have $90 \%$ power to detect a $2.9 \%$ reduction in 90 day mortality in the study population; this is less than the reduction in mortality reported in database studies. These calculations assumed a base mortality rate of $23 \%$ and $2 \%$ of patients lost to follow-up. Following the advent of the coronavirus disease 2019 (COVID-19) pandemic, the study Management Committee, in conjunction with the study sponsor (the George Institute for Global Health, Australia) faced suspension of non-COVID-19 clinical research in many of the study hospitals and significant uncertainty over prospects for future recruitment. The Australian National Health and Medical Research Council, the major study funder, was approached for further funding to keep the study running for longer but did not have the funds or a mechanism to allocate additional funding. Consequently, power calculations were undertaken to assess the impact of ceasing recruitment on 31 December 2020, a date 60 days before the expiry of the current batch of study fluids. Both the initial and revised power calculations are detailed in Table 3. Assuming $2 \%$ of patients lost to follow-up, the revised plan is to include $\sim 5000$ patients, which provides $90 \%$ power to detect a hypothesised absolute difference of 3.8 percentage points (ie, one percentage point more than originally intended), and $80 \%$ power to detect a hypothesised absolute between-group difference of 3.3 percentage points (ie, 0.4 percentage points more than originally intended) at $\alpha=0.05$.

\section{Statistical analysis}

\section{Analysis principles}

Analyses will be conducted on an intention-to-treat basis. The intention-to-treat population is all patients randomised regardless of whether they receive study treatment, and includes patients for whom there are no data available due to absence or revocation of consent. To comply with relevant laws, data for which consent was not obtained or was withdrawn will be excluded from the analyses and subject to sensitivity analyses using missing data imputations.

A sensitivity analysis of the primary outcome will be conducted after sequentially (and cumulatively) excluding the following patients:

- patients who received $500 \mathrm{~mL}$ or more of the study fluid (Plasma-Lyte 148 or $0.9 \%$ saline) as an open label fluid before enrolment when they were assigned to the other fluid; and

- patients who received $500 \mathrm{~mL}$ or more of the study fluid (Plasma-Lyte 148 or $0.9 \%$ saline) as an open label fluid in the ICU (post-randomisation) when they were assigned to the other fluid. 


\section{ORIGINAL ARTICLES}

Table 3. Initial and revised power calculations

\begin{tabular}{lccccc} 
Power & $\boldsymbol{N}$ & $\begin{array}{c}\text { Mortality rate for } \\
\mathbf{0 . 9 \%} \text { saline }\end{array}$ & $\begin{array}{c}\text { Mortality rate for } \\
\text { Plasma-Lyte 148 }\end{array}$ & Difference & $\boldsymbol{\alpha}$ \\
\hline $90 \%$ & 8596 & $23.00 \%$ & $20.13 \%$ & $-2.87 \%$ & 0.05 \\
$80 \%$ & 8596 & $23.00 \%$ & $20.51 \%$ & $-2.49 \%$ & 0.05 \\
$90 \%$ & 4900 & $23.00 \%$ & $19.22 \%$ & $-3.78 \%$ & 0.05 \\
$80 \%$ & 4900 & $23.00 \%$ & $19.72 \%$ & $-3.28 \%$ & 0.05 \\
\hline
\end{tabular}

The figure will consist of two (one plot per arm) stacked bar charts with one bar per day and, within each bar, the proportion of patients in each category (summing to $100 \%$ ).

\section{Participant characteristics and baseline comparisons}

Baseline characteristics (Table 4)

All tests are to be two-sided, with a nominal level of $\alpha$ set at $5 \%$. Analyses of the primary outcome (all-cause mortality at 90 days) will be unadjusted for multiplicity; however, we will control the family-wise error rate across secondary outcomes using a Holm-Bonferroni correction. ${ }^{8}$ No other multiplicity adjustment will be applied.

\section{Participant flow}

The flow of patients through the trial will be displayed in a CONSORT $^{9}$ (Consolidated Standards of Reporting Trials) diagram. The report will include the number of screened patients who met study inclusion criteria and the number of patients who were included, and reasons for exclusion of non-included patients. A separate figure will describe their consenting status.

In addition, we will create a chart showing, for each day between days 1-90, the proportion of patients in each arm who fall into one these five categories:

- alive and in the ICU;

- discharged from the ICU but still in hospital;

- discharged alive from hospital;

- dead; and

- unknown status. will be presented by treatment group. Discrete variables will be summarised by frequencies and percentages. Percentages will be calculated according to the number of patients for whom data are available. Continuous variables will be summarised using mean and standard deviation (SD) together with the median and interquartile range (IQR).

\section{Analysis of daily data}

Fluid details, laboratory values and measures of organ dysfunction collected daily will be summarised using descriptive statistics (mean [SD], median [IQR], minimum and maximum for continuous variables, or number and percentages for categorical variables) and plots (mean plots or bar charts) per day. For continuous variables, the overall mean per treatment arm and overall difference (and 95\% confidence interval $[\mathrm{Cl}]$ ) between treatment arms will be calculated using a repeated-measure linear mixed model with a fixed effect of treatment, a fixed categorical effect of time (study day), a fixed interaction between treatment and time, a fixed continuous effect of the baseline value, a random site effect (to model within-site correlations), and a random patient effect (to model within-patient correlations). Binary and ordinal variables will be analysed

\section{Table 4. Baseline data}

- Demographic characteristics: age (years), sex and weight $(\mathrm{kg})$

- ICU admission details: source and diagnosis

- Severity of illness score (APACHE II)

- Sepsis: presence of infection (yes/no), infection sites and organisms, SIRS criteria

- Renal function before/at time of randomisation: last serum creatinine value and renal replacement therapy

- Fluids received within 24 hours before randomisation

- Clinical data (last value before randomisation): heart rate, mean arterial pressure, central venous pressure, pH, base excess, serum lactate, potassium, chloride, haemoglobin level

- Mechanical ventilation (yes/no)

- Renal replacement therapy (yes/no)

- Organ dysfunction (most deranged value/score within 24 hours before randomisation): respiratory SOFA score, cardiovascular SOFA score, highest creatinine value, highest bilirubin value, lowest platelet count, worst GCS 


\section{ORIGINAL ARTICLES}

\section{Table 5. Subgroups}

- Age (<65 $v \geq 65$ years)

- Sex (male $v$ female)

- Presence $v$ absence of kidney injury (defined as creatinine concentration at least $>1.5$ times above the upper limit of the reference range for the local laboratory)

- Presence $v$ absence of sepsis (defined using 2016 SOFA-based criteria)

- Admission to the ICU directly after surgery or not

- Low $v$ high severity of illness (defined by APACHE II score $<25$ or $\geq 25$ )

APACHE = Acute Physiology and Chronic Health Evaluation; ICU = intensive care unit; SOFA = Sequential Organ Failure Assessment.

using a similar modelling approach but using logistic regression and ordinal logistic regression, respectively, in place of linear regression and with the between treatment differences estimated as odds ratio (OR) and $95 \% \mathrm{CI}$. While these metrics are subject to competing risks due to patients who die or are discharged from the ICU before Day 7, these analyses are aimed at describing the intervention process rather than estimate its effect and, therefore, we will not adjust for competing risks or impute for missing data.

\section{Analysis of the primary outcome}

The primary analysis will be conducted without imputation of missing data. The primary endpoint is the proportion of participants dead at 90 days. To account for stratification by site and maximise power, ${ }^{10}$ the main analysis will be performed using logistic regression, with treatment allocation as a fixed effect and site as a random effect. ${ }^{11}$ The effect of the intervention will be presented as the OR of death and $95 \% \mathrm{Cl}$. Crude proportions by treatment arm will also be reported with an unadjusted $\mathrm{OR}$ and $95 \% \mathrm{Cl}$ and a $\chi^{2}$ test $P$ value. For ease of interpretation, risk difference and $95 \% \mathrm{Cls}$ will also be presented. The adjusted OR and $95 \% \mathrm{Cl}$ will be converted to an adjusted risk difference and $95 \% \mathrm{Cl}$ using the Hummel and Wiseman method described by Reeve. ${ }^{12}$

Adjusted analyses will be performed by adding the following covariates to the main logistic regression model: sex, Acute Physiology and Chronic Health Evaluation (APACHE) II score at randomisation (as a continuous variable), presence of sepsis, and source of admission (postoperative $v$ other). The adjusted treatment effect will be reported as the adjusted OR and $95 \% \mathrm{Cl}$.

\section{Subgroup analyses}

Six pre-specified subgroup analyses (Table 5) will be carried out irrespective of whether there is a significant treatment effect on the primary outcome. The analysis for each subgroup will be performed by adding the subgroup variable as well as its interaction with the intervention as fixed effects to the main logistic regression model. Within each subgroup, summary measures will include raw counts and percentages within each treatment arm, as well as the OR for treatment effect with a $95 \% \mathrm{Cl}$. The results will be displayed on a forest plot including the $P$ value for heterogeneity corresponding to the interaction term between the intervention and the subgroup variable.

\section{Treatment of missing data}

If more than $3 \%$ of patients from the intention-to-treat population are excluded from the analysis of death at Day 90 due to missing data, missing data will be imputed using "worst-best" and "best-worst" case scenarios. In the "worst-best" scenario, the "worst" outcome (ie, dead at Day 90) will be assigned to all patients missing the outcome in one treatment group, and the "best" outcome (ie, alive at Day 90) will be assigned to all patients missing the outcome in the other treatment group. The "best-worst" scenario corresponds to the reverse assignment of outcomes. If these two extreme scenarios lead to the same conclusions, no further imputation of missing data will be performed. In case of inconsistent conclusions; that is, where one scenario leads to a statistically significant difference and not the other or where the two are significant but in different directions, we will further explore the impact of missing data by performing multiple imputations using fully conditional specification. ${ }^{13}$ The imputation model will include death at 90 days, the randomised treatment arm, study site, all baseline data and all variables collected during Days 1-7. Binary variables (eg, vital status at 90 days) will be imputed using an ordinal logistic model, categorical variables using a discriminant function method and continuous variables using linear regression. One-hundred sets of imputed data will be created and analysed using the methods described for the analysis of the primary end point. 


\section{ORIGINAL ARTICLES}

\section{Other analyses of mortality}

The primary mortality analysis will be replicated to compare the proportions of patients who died at ICU discharge, hospital discharge, and at Day 28. For these analyses, no adjusted or subgroup analyses will be performed. Causes (proximate and underlying) and places of death (by Day 90) will be categorised and the distribution will be compared between the two treatment arms using a $\chi^{2}$ test. An analysis of time to death by treatment group will be censored at Day 90 or at the time when the patient was last known to be alive, whichever occurs earlier. A Kaplan-Meier plot will be used to describe survival rates and derive median and quartiles of time to death. Differences in survival will be tested using a Cox model with a random site effect (ie, using a shared-parameter frailty model). ${ }^{14}$

\section{Analyses of other secondary outcomes}

Serum creatinine. The overall mean serum creatinine value per treatment arm and overall difference (and 95\% Cl) between treatment arms over Days 1-7 will be calculated using a repeated-measure linear mixed model, as described in the section on analysis of daily data. In addition, peak creatinine level and maximum post-randomisation increase in creatinine level will be analysed using an analysis of covariance adjusted on the baseline creatinine value and including a fixed effect of treatment and a random effect of site. These analyses will be performed without imputing missing data; however, to assess the potential impact of informative missing data due to early discharge or death, we plan to describe creatinine values between Days 1-7 separately according to when patients died or were discharged alive. Fourteen profiles, or strata, will thus be created according to the day when the patient died or was discharged (Days 1-7) and separately for those who died versus those who were discharged alive $(\times 2)$. For each of these 14 profiles, we will describe the daily creatinine values using non-parametric cubic splines. In case of a clear indication that the creatinine trajectories differ according to the profile, we will consider re-running the repeatedmeasure linear model using a pattern-mixture approach adjusted by profile.

Durations and time to discharge. The hospital length of stay, ICU length of stay, duration of mechanical ventilation, and duration of treatment with vasoactive drugs will be analysed as the number of days alive and free of outcome (eg, days alive and free of mechanical ventilation or days alive and free/outside of ICU). Days alive and free of outcome will be calculated between randomisation and Day 90 and will therefore have values comprised between 0 and 90 days. They will be summarised using means (SDs), median (IQRs), minimum and maximum and compared between treatment groups using a linear regression with a fixed effect of the treatment group and a random effect of site. As a sensitivity analysis, we will allocate zero "free days" to patients who die by Day 90 . Mechanical ventilation and treatment with vasoactive drugs are only expected to occur while in the ICU. Therefore, once discharged from the ICU, patients will be assumed to be free of respiratory support and vasopressors. While in the ICU, missing daily values (eg, unknown mechanical ventilation status) will be handled as follows:

- Step 1. For intermittent missing values (ie, missing values surrounded by non-missing values both before and after), we will replace the missing value with the closest (in time) non-missing value. In case the time intervals before and after are the same, the missing value will be replaced with the most pessimistic value of the two, meaning still in the ICU or hospital or still receiving the treatment of interest.

- Step 2. For missing values following the baseline assessment (ie, occurring on Day 1 onwards), the mechanical ventilation status at the time of randomisation and the most deranged cardiovascular score within 24 hours before randomisation will be used to guide imputation. In case of missing baseline values, the patient will be assumed to be free of the treatment of interest at baseline. After imputation of the baseline value, missing values will be imputed by following Step 1 above.

- Step 3. For missing values preceding ICU discharge, the patient will be assumed to be free of outcome on the day of discharge if discharged alive and not if they died within a day of ICU discharge. After imputation of the value on the day of discharge, missing values will be imputed by following Step 1 above.

Time to discharge alive from index ICU and time to discharge alive from index hospital admission will be summarised using cumulative incidence functions treating mortality as a competing risk. Medians and quartiles of time to discharge will be obtained from the cumulative incidence functions. The effect of the intervention will be estimated as the hazard ratio (Plasma-Lyte 148 divided by $0.9 \%$ saline) and $95 \% \mathrm{Cl}$ obtained from a Cox model of the cause-specific hazard, which estimates the risk of discharge in subjects who are still alive and have not yet been discharged. ${ }^{15}$ To model potential within-site correlations due to stratification, we will use a shared-parameter frailty Cox model with a fixed effect of treatment and a random site effect. ${ }^{14}$

\section{New treatment with renal replacement therapy and vasoactive drugs. Comparison of proportions of patients newly treated with renal replacement therapy and of patients treated with vasoactive drugs will be summarised by treatment arm and compared using a logistics regression analysis analogous to that used for the primary outcome.}

Quality of life. The information obtained from the Fivelevel EuroQol five dimensions (EQ-5D-5L) questionnaire will 


\section{ORIGINAL ARTICLES}

be used to conduct a cost-utility analysis at 6 months after randomisation and to report quality of life by treatment group and by subgroups. The cost utility analysis forms part of an extended program of health economic and outcomes research to be conducted after publication of the main trial findings.

Safety outcomes. Adverse drug reactions deemed possibly, probably or definitely related to study treatment as determined by the treating physician at site will be summarised as the number and proportion of patients experiencing at least one event. These will be summarised by category of event and overall numbers of events. In addition to the number of patients with at least one event, we will report the total number of events. Proportions of patients with adverse drug reactions will be compared between treatment arms using Fisher exact test, both overall and by category. This will be repeated for serious adverse drug reactions. A listing of all adverse drug reactions will be reported in an Online Appendix.

Acknowledgements: A series of mock tables and figures is available online with the extended version of the PLUS study Statistical Analysis Plan at https://osf.io/8gk3n. Our study is funded by the Australian National Health and Medical Research Council (Project Grant 1101765) and the Health Research Council of New Zealand (Project Grant 16/09). Baxter Healthcare will provide the blinded study fluids for the trial and will manage the warehousing and distribution of the fluids. The data will be managed, analysed and reported independent of Baxter Healthcare and the funding agencies. The full list of participating sites and investigators is available in the Online Appendix.

\section{Competing interests}

None declared.

\section{Author details}

Laurent Billot 1,2

Rinaldo Bellomo $3,4,5,6$

Martin Gallagher 2,7,8,9

David Gattas ${ }^{10,11}$

Naomi E Hammond 2,12,13

Diane Mackle ${ }^{14,15}$

Sharon Micallef ${ }^{12}$

John Myburgh2,12,16

Leanlove Navarra14,15

Manoj Saxena ${ }^{12,17}$

Colman Taylor ${ }^{2,12}$

Paul J Young ${ }^{14,15}$

Simon Finfer $2,12,13,18$

On behalf of the PLUS Study investigators and the ANZICS

Clinical Trials Group
1 Statistics Division, George Institute for Global Health, Sydney, NSW, Australia.

2 Faculty of Medicine, University of New South Wales, Sydney, NSW, Australia.

3 Intensive Care Unit, Austin Hospital, Melbourne, VIC, Australia.

4 Department of Intensive Care, Royal Melbourne Hospital, Melbourne, VIC, Australia.

5 Centre for Integrated Critical Care, University of Melbourne, Melbourne, VIC, Australia.

6 Australian and New Zealand Intensive Care Research Centre, School of Public health and Preventive Medicine, Monash University, Melbourne, VIC, Australia.

7 Concord Clinical School, University of Sydney, Sydney, NSW, Australia.

8 Renal and Metabolic Division, George Institute for Global Health, Sydney, NSW, Australia.

9 Concord Repatriation General Hospital, Sydney, NSW, Australia

10 Intensive Care Unit, Royal Prince Alfred Hospital, Sydney, NSW, Australia.

11 Central Clinical School, School, University of Sydney, Sydney, NSW, Australia

12 Critical Care Division, George Institute for Global Health, Sydney, NSW, Australia.

13 Malcolm Fisher Department of Intensive Care, Royal North Shore Hospital, Sydney, NSW, Australia.

14 Medical Research Institute of New Zealand, Wellington, New Zealand.

15 Intensive Care Unit, Wellington Hospital, Wellington, New Zealand.

16 Intensive Care Unit, St George Hospital, Sydney, NSW, Australia.

17 Intensive Care Unit, Bankstown Hospital, Sydney, NSW, Australia.

18 School of Public Health, Imperial College, London; United Kingdom.

Correspondence: Ibillot@georgeinstitute.org

\section{References}

1 Hammond NE, Bellomo R, Gallagher M, et al. The Plasma-Lyte 148 v Saline (PLUS) study protocol: a multicentre, randomised controlled trial of the effect of intensive care fluid therapy on mortality. Crit Care Resusc 2017; 19: 239-46.

2 Finfer S, Bellomo R. Why publish statistical analysis plans? Crit Care Resusc 2009; 11: 5-6.

3 Myburgh J, Li Q, Heritier S, Dan A, Glass P. Statistical analysis plan for the Crystalloid Versus Hydroxyethyl Starch Trial (CHEST). Crit Care Resusc 2012; 14: 44-52.

4 TARGET Investigators on behalf of the Australian and New Zealand Intensive Care Society Clinical Trials Group. Statistical analysis plan for the Augmented versus Routine Approach to Giving Energy Trial (TARGET). Crit Care Resusc 2018; 20: 15-21.

5 Mackle DM, Bailey MJ, Beasley RW, et al. Protocol summary and statistical analysis plan for the intensive care unit randomised 


\section{ORIGINAL ARTICLES}

trial comparing two approaches to oxygen therapy (ICU-ROX). Crit Care Resusc 2018; 20: 22-32.

6 Young PJ, Delaney AP, Dulhunty JM, Venkatesh B. Critical care statistical analysis plans. In reply. Crit Care Resusc 2014; 16: 76-7.

7 Billot L, Finfer S; PLUS Management Committee. The PLUS study statistical analysis plan; version 1.0 (19 Aug 2020). https://osf.io/8gk3n (viewed Sept 2020).

8 Holm S. A simple sequentially rejective multiple test procedure. Scand J Stat 1979; 6: 65-70.

9 Moher D, Schulz KF, Altman DG. The CONSORT statement: revised recommendations for improving the quality of reports of parallel-group randomized trials. JAMA 2001; 285: 1987-91.

10 Kahan BC, Morris TP. Improper analysis of trials randomised using stratified blocks or minimisation. Stat Med 2012; 31: 328-40.
11 Kahan BC. Accounting for centre-effects in multicentre trials with a binary outcome - when, why, and how? BMC Med Res Methodol 2014; 14: 20.

12 Reeve R. Confidence interval of difference of proportions in logistic regression in presence of covariates. Stat Methods Med Res 2018; 27: 451-65.

13 van Buuren S. Multiple imputation of discrete and continuous data by fully conditional specification. Stat Methods Med Res 2007; 16: 219-42.

14 Ripatti S, Palmgren J. Estimation of multivariate frailty models using penalized partial likelihood. Biometrics 2000; 56: 101622.

15 Austin PC, Lee DS, Fine JP. Introduction to the analysis of survival data in the presence of competing risks. Circulation 2016; 133: 601-9. 